\title{
A general strategy for high-throughput experimental screening of promising bulk thermoelectric materials
}

\author{
Shiyang $\mathrm{He}^{1 \dagger}$, Yang Yang ${ }^{2 \dagger}$, Zhili $\mathrm{Li}^{1}$, Jiye Zhang ${ }^{1}$, Chenyang Wang ${ }^{1}$, Wenqing Zhang ${ }^{3}$ and \\ Jun Luo ${ }^{1,4^{*}}$
}

\begin{abstract}
High-throughput (HTP) experiments play key roles in accelerating the discovery of advanced materials, but the HTP preparation and characterization, especially for bulk samples, are extremely difficult. In this work, we developed a novel and general strategy for HTP screening of high-performance bulk thermoelectric materials. The performed fullchain HTP experiments cover rapid synthesis of the bulk sample with quasi-continuous composition, microarea phase identification and structure analysis, and measurement of the spatial distribution of the sample composition, electrical and thermal transport properties. According to our experiments, bulk $\mathrm{Bi}_{2-x} \mathrm{Sb}_{x} \mathrm{Te}_{3}(x=1-2)$ and $\mathrm{Bi}_{2} \mathrm{Te}_{3-x} \mathrm{Se}_{x}(x=0-1.5)$ samples with quasi-continuous compositions have been rapidly fabricated by this HTP method. The target thermoelectric materials with the best $\mathrm{Sb} / \mathrm{Bi}$ and $\mathrm{Te} / \mathrm{Se}$ ratios are successfully screened out based on subsequent HTP characterization results, demonstrating that this HTP technique is effective in speeding up the exploration of novel high-performance thermoelectric materials.
\end{abstract}

Keywords: high-throughput experimental screening, thermoelectric materials, electrical transport properties, thermal transport properties, $(\mathrm{Bi}, \mathrm{Sb})_{2}(\mathrm{Te}, \mathrm{Se})_{3}$

\section{INTRODUCTION}

Searching for new materials is of great significance due to the persistent demand for advanced materials of modern society, but the efficiency of material discovery has long been limited by the traditional Edisonian trial and error approach [1-3]. Recently, high-throughput (HTP) techniques have attracted intensive efforts owing to their abilities in speeding up the research and development of novel materials [4-7]. HTP prediction based on theoretical calculation [8] and machine learning [9] has made remarkable progress and achieved great success in a variety of materials [10-13]. For example, simultaneous advances in theoretical calculation, integrated database and machine learning have initiated new revolutions of materials development in the field of superconducting and fluorescent materials [14-16]. However, HTP experiments, particularly for bulk samples, have lagged behind [17]. HTP experiments are important for the searching of new materials because they can not only verify the result of HTP predication, but also help to calibrate and enrich computational models and material database by providing experimentally measured data, which further promote the development of HTP predication in turn $[6,18]$. Thus, HTP experiments are capable of launching material design into a new era [19].

Based on the characteristics of composition distribution, HTP sample fabrication can be classified into two types: material gene chip technique and continuously gradient component preparation technology [2]. The material gene chip technique is a 'parallel' method to fabricate samples in the form of thin-film by the combination of designed masks or moving shutters to build up layers, which results in a net variation in composition $[20,21]$. Thus, it allows hundreds of individually separated thin-film samples to be synthesized in one substrate. However, this method intends to miss certain mutating performance due to the characteristics of discrete composition distribution [22]. Moreover, the relevant prop-

\footnotetext{
${ }^{1}$ School of Materials Science and Engineering, Shanghai University, Shanghai 200444, China

${ }^{2}$ School of Mechatronic Engineering and Automation, Shanghai University, Shanghai 200444, China

${ }^{3}$ Department of Physics, Shenzhen Institute for Quantum Science and Engineering, Guangdong Provincial Key-Lab for Computational Science and Materials Design, Southern University of Science and Technology, Shenzhen 518055, China

${ }^{4}$ Materials Genome Institute, Shanghai University, Shanghai 200444, China

${ }^{\dagger}$ These authors contributed equally to this work.

* Corresponding author (email: junluo@shu.edu.cn)
} 
erties obtained from the thin-film samples may not directly map to the bulk material due to the size effect. The continuously gradient component preparation technology is mainly concentrating on the bulk materials $[23,24]$ and usually adopted to fabricate multiple diffusion couples [25-28]. By virtue of the creation of composition gradients through long-term annealing, this method enables the generation of plenty of phases and compositions, which are efficient mappings for phase diagrams and physical properties in preceding studies [29,30]. Although the diffusion couple method can be regarded as a HTP experimental method for bulk samples, it is a great challenge for sample performance characterization because the composition variation is restricted in a pretty small area [31,32]. Therefore, it is urgently needed to develop a HTP experimental method with wide applicability for bulk samples.

In this work, a novel and general strategy for HTP screening of high-performance bulk thermoelectric materials is developed based on a continuously gradient component technique. The bulk sample with quasi-continuous composition is rapidly fabricated by spark plasma sintering (SPS) and annealing the raw material bar with gradient composition which is automatically prepared by a self-developed automatic powder feeding system. The rapid screening of the target material is realized by constructing the structure-composition-property relationship of the bulk sample with quasi-continuous composition according to the HTP characterization results of its spatial distribution of the structure, composition, electrical and thermal transport properties. The effectiveness and practicality of our HTP strategy are established by the HTP experiments on the state-of-the-art $\mathrm{Bi}_{2-x} \mathrm{Sb}_{x} \mathrm{Te}_{3} /$ $\mathrm{Bi}_{2} \mathrm{Te}_{3-x} \mathrm{Se}_{x}$ thermoelectric systems, demonstrating that this HTP technique can be utilized to speed up the discovery of novel high-performance thermoelectric materials. With this HTP strategy, the time spent in finding the target composition, especially the labor time, is significantly reduced (Fig. 1), showing the great potential of HTP experimental screening for advanced thermoelectric materials.

\section{EXPERIMENTAL SECTION}

\section{Synthesis of HTP samples}

The n-type $\mathrm{Bi}_{2} \mathrm{Te}_{3}$ was prepared by a ball-milling method. High-purity bismuth and tellurium pieces (Bi, 99.99\%; $\mathrm{Te}, 99.9 \%)$ were weighed according to the Te-rich composition ratio $(\mathrm{Bi}: \mathrm{Te}=2: 3.02)$, which were then loaded into a stainless-steel ball-milling jar in a glove box under an argon atmosphere. All element pieces were ball-milled for $8 \mathrm{~h}$ to obtain pure $\mathrm{Bi}_{2} \mathrm{Te}_{3}$ powders.

The ingots of p-type $\mathrm{Bi}_{2} \mathrm{Te}_{3}$, p-type $\mathrm{Sb}_{2} \mathrm{Te}_{3}$ and n-type $\mathrm{Bi}_{2} \mathrm{Se}_{3}$ were prepared by a conventional melting route. High-purity stoichiometric elements ( $\mathrm{Bi}, 99.99 \%$; Te, 99.9\%; Sb, 99.99\%; Se, 99.9\%) were weighed and mixed in graphite crucibles, sealed in quartz tubes under vacuum and then heated at high temperature (the heating temperature for $\mathrm{Sb}_{2} \mathrm{Te}_{3} / \mathrm{Bi}_{2} \mathrm{Te}_{3}$ and $\mathrm{Bi}_{2} \mathrm{Se}_{3}$ are 1173 and $823 \mathrm{~K}$, respectively). The melting and reaction duration were $72 \mathrm{~h}$ to ensure complete homogeneous. Subsequently, the quartz tubes were naturally cooled to room temperature in the furnace. The obtained ingots were milled in stainless-steel ball-milling jar for $100 \mathrm{~min}$. The procedure of milling was not intended to cause mechanical alloying but only reduced the particle size of powders for efficient diffusion.

The cylinder-like HTP samples with gradient compositions were automatically prepared by the self-developed automatic powder feeding system and then densified by spark plasma sintering in the vacuum at $723 \mathrm{~K}$ for $30 \mathrm{~min}$. All the samples were annealed at $673 \mathrm{~K}$ for different times in quartz ampoules filled with $0.5 \mathrm{~Pa}$ of Ar. The obtained samples were directly cut into several thin

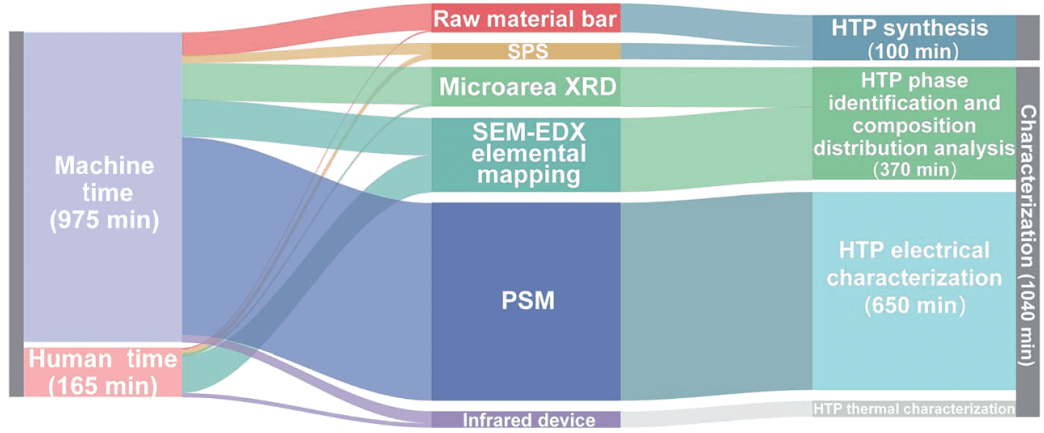

Figure 1 Sankey diagram showing the time taken by each process of the HTP experiment. 
slabs by a diamond saw for different characterizations.

\section{Sample characterization}

Phase identification and structure analysis were performed on precursor materials by a conventional X-ray powder diffraction (XRD) technique. Microarea XRD analysis was applied on the HTP thin slab by a Rigaku SmartLab-II diffractometer with $\mathrm{Cu}$ Ka radiation $(40 \mathrm{kV}$ $\times 30 \mathrm{~mA}$ ) and the measuring interval was set at $400 \mu \mathrm{m}$. Sample compositions throughout the sample were performed by a scanning electron microscope (SEM, Gemini 300, Zeiss) equipped with an energy dispersive X-ray detector (EDX, Oxford Instrument). Spatially resolved room-temperature Seebeck coefficients were scanned along the entire composition gradient by a potential Seebeck microprobe (PSM II, Panco Ltd., Germany) with a grid point spacing of $50 \mu \mathrm{m}$. The thermal properties for the HTP thin slab were obtained by our homemade thermal performance infrared screening system (detailed description of the measurement setup can be found in Reference [17]).

\section{RESULTS AND DISCUSSION}

To enable the HTP screening of target materials, the structure-composition-property relationship of the bulk sample with continuous composition must be constructed. Thus, our HTP screening strategy is divided into three procedures as shown in Fig. 2. Firstly, the HTP sample with quasi-continuous composition is rapidly fabricated. Secondly, the structure and composition distribution of the HTP sample are fast identified. Finally, the property distribution including electrical and thermal properties is quickly characterized. In this work, the wellknown $\mathrm{Bi}_{2-x} \mathrm{Sb}_{x} \mathrm{Te}_{3} / \mathrm{Bi}_{2} \mathrm{Te}_{3-x} \mathrm{Se}_{x}$ alloys have been used as the prototype materials to provide a demonstration and proof of concept for our HTP strategy.

In the first step, the fabrication process of the HTP sample contains the preparation of gradient cylinder and subsequent annealing treatment. For the convenience of fabricating HTP bulk sample with quasi-continuous composition, the binary p- and n-type $\mathrm{Bi}_{2} \mathrm{Te}_{3}$, p-type $\mathrm{Sb}_{2} \mathrm{Te}_{3}$ and $\mathrm{n}$-type $\mathrm{Bi}_{2} \mathrm{Se}_{3}$ compounds were pre-synthesized as the precursors by a conventional melting or ballmilling method (see Fig. S1 for the XRD patterns of these precursors). The HTP $\mathrm{Bi}_{2-x} \mathrm{Sb}_{x} \mathrm{Te}_{3}$ sample was made from the p-type $\mathrm{Bi}_{2} \mathrm{Te}_{3}$ and p-type $\mathrm{Sb}_{2} \mathrm{Te}_{3}$ precursors, while the HTP $\mathrm{Bi}_{2} \mathrm{Te}_{3-x} \mathrm{Se}_{x}$ sample was made from the n-type $\mathrm{Bi}_{2} \mathrm{Te}_{3}$ and n-type $\mathrm{Bi}_{2} \mathrm{Se}_{3}$ precursors. The multi-layer raw material bar shown in Fig. 2 was fabricated by automatically weighing, mixing and loading the precursor powders into a graphite die using the homemade automatic powder feeding system (the detailed setup and function of the device can be found in Reference [28]) according to the composition gradient $\left(\mathrm{Bi}_{2-x} \mathrm{Sb}_{x} \mathrm{Te}_{3}\right.$ with $x=0,0.2,0.4, \ldots$, $1 ; \mathrm{Bi}_{2} \mathrm{Te}_{3-x} \mathrm{Se}_{x}$ with $\left.x=0,0.3,0.6, \ldots, 1.5\right)$. The highdensity cylinder-like sample with quasi-continuous composition was obtained by SPS sintering and subsequent annealing in sealed quartz tubes in an argon atmosphere. The obtained sample was directly cut into several thin slabs by a diamond saw for various characterizations.

To reveal the phase composition and crystal structure

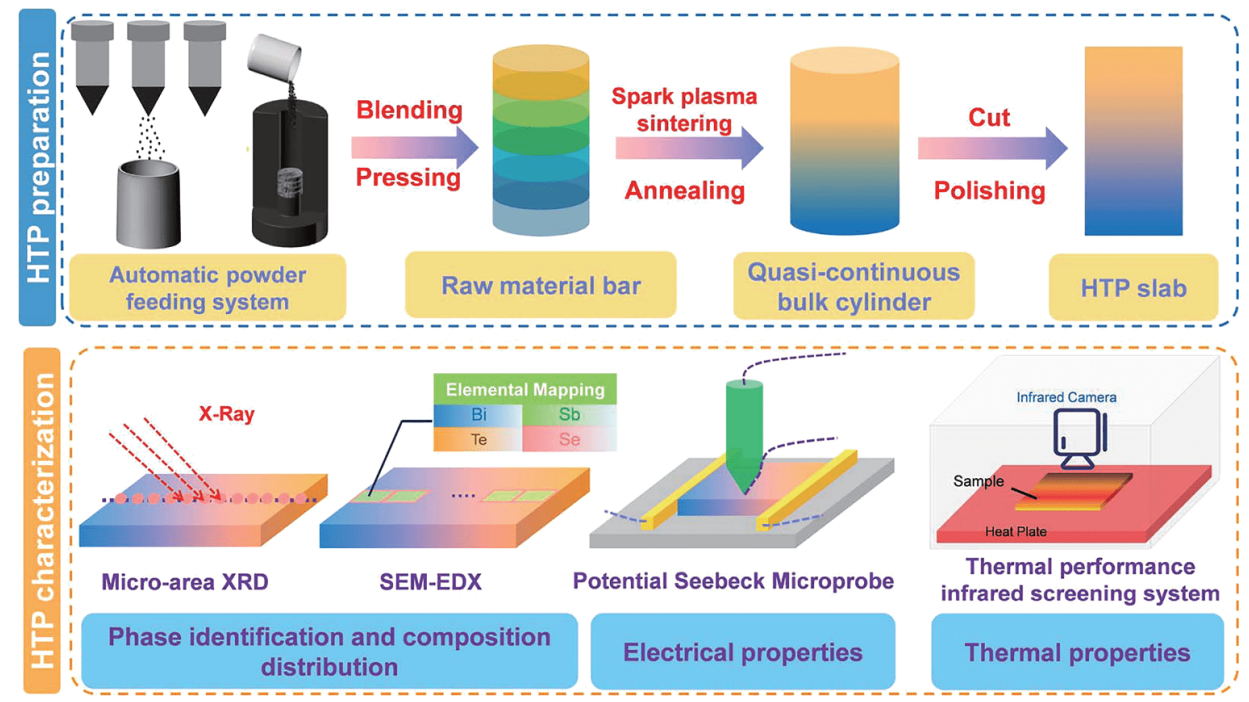

Figure 2 Schematic diagram for our HTP experimental screening. 
of the HTP samples, the thin slab was analyzed by the microarea XRD. The total length of the $\mathrm{Bi}_{2-x} \mathrm{Sb}_{x} \mathrm{Te}_{3}(x=$ $1-2)$ slab is about $6 \mathrm{~mm}$ along the composition gradient, and 14 micro regions were selected for microarea XRD analysis in an interval of $400 \mu \mathrm{m}$ (Fig. 3b and Fig. S2b). For both as-prepared and annealed for 3 days samples, the XRD patterns of all regions can be indexed to the rhombohedral structure with the space group $R \overline{3} m$ and no obvious impurities are observed (see Fig. $3 \mathrm{a}$ and Fig. S2a), indicating that the $\mathrm{Bi}_{2-x} \mathrm{Sb}_{x} \mathrm{Te}_{3}$ solid solution is formed. Further analysis reveals that the diffraction peaks around $28.5^{\circ}$ shift slightly to low angles and show almost linear change from region 1 to 14 . Moreover, as shown in Fig. $3 c$, the lattice parameter $c$ increases gradually from 4.26 to $4.34 \AA$, and the linear coefficient of lattice parameter is about 0.95 along the increasing direction of $\mathrm{Bi}$ content. In comparison with the lattice parameters of the as-prepared sample (Fig. S2c), the annealed sample exhibits much stronger linear relationship (the linear coefficient of the as-prepared sample is below 0.89). Similarly, both the as-prepared and annealed $\mathrm{Bi}_{2} \mathrm{Te}_{3-x} \mathrm{Se}_{x}$ HTP slabs form solid solutions, and the annealed HTP slab also shows a higher linear coefficient of lattice parameter (above 0.94) than the as-prepared HTP slab (Figs S3 and S4).

The composition distribution of the HTP slab was further investigated by the SEM-EDX elemental mapping. The polished area of the HTP thin slab is about $6 \mathrm{~mm}$ along the composition gradient, which is too large for a one-time measurement using the SEM-EDX. Thus, the entire sample was labeled with multiple regions, and each region was analyzed with EDX to determine the composition distribution separately. Fig. 4 shows the composition distribution of the $\mathrm{Bi}_{2-x} \mathrm{Sb}_{x} \mathrm{Te}_{3}$ thin slab with different annealing times. For the as-prepared sample, obvious Bi-rich phases can be observed in the elemental mappings shown in Fig. 4a. After annealing, the Bi-rich phases disappear and quasi-continuous composition distribution is achieved in the entire thin-slab sample (Fig. 4b). The linear coefficient of composition distribution increases with the annealing time, which reaches 0.99 for the sample annealed for 3 days. The annealed $\mathrm{Bi}_{2} \mathrm{Te}_{3-x} \mathrm{Se}_{x}$ thin slab also shows quasi-continuous composition, indicating that annealing process is effective on controlling the composition diffusion (Fig. S5).

According to the results of microarea XRD and SEMEDX measurements, bulk $\mathrm{Bi}_{2-x} \mathrm{Sb}_{x} \mathrm{Te}_{3} / \mathrm{Bi}_{2} \mathrm{Te}_{3-x} \mathrm{Se}_{x}$ samples with quasi-continuous compositions have been successfully fabricated by our HTP preparation technique, which provides the appropriate specimen for the subsequent HTP screening of electrical and thermal transport properties. As shown in the left panel of Fig. $5 \mathrm{a}-\mathrm{c}$, the Seebeck
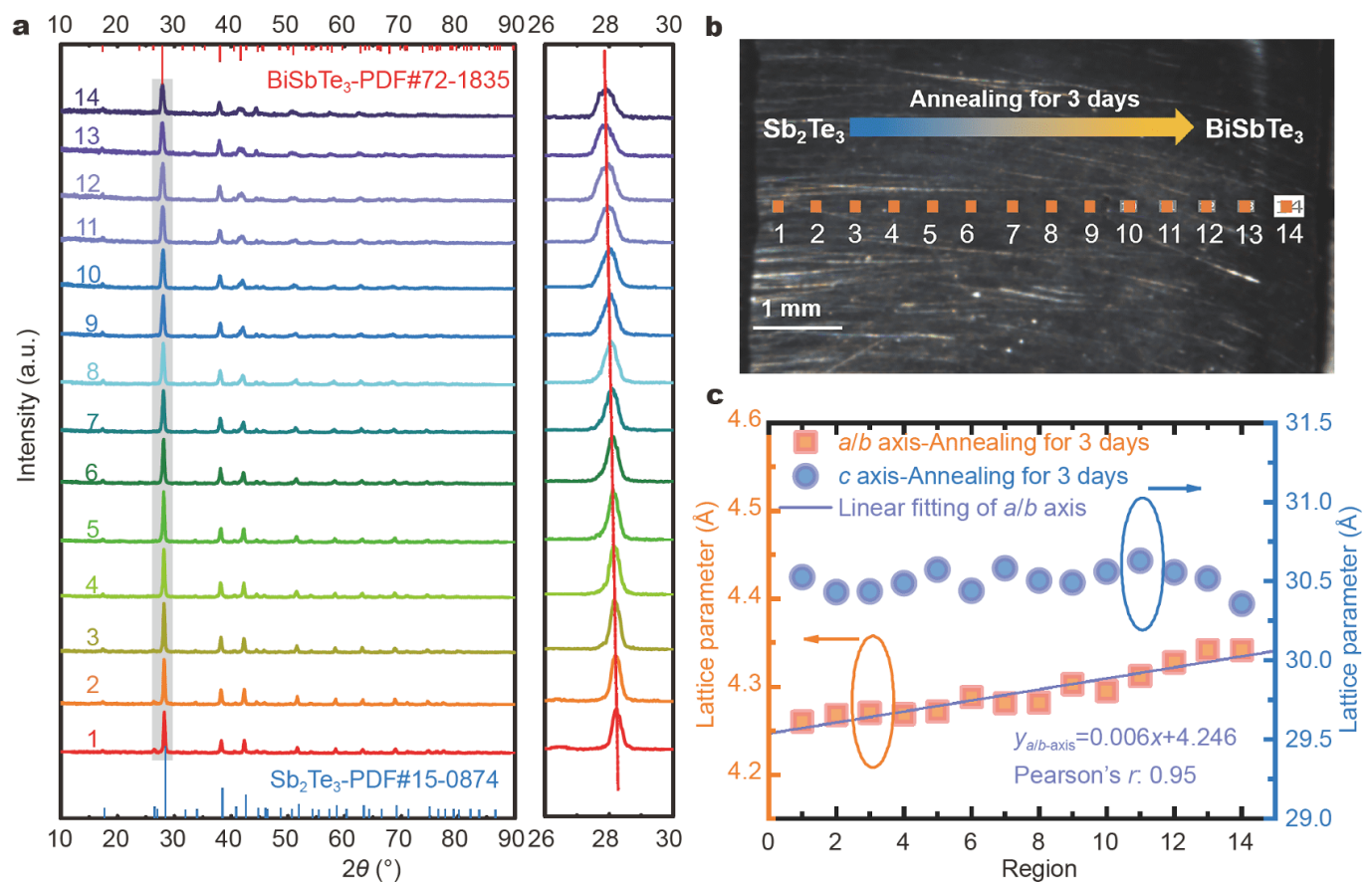

Figure 3 Phase identification and structure analysis of the annealed $\mathrm{Bi}_{2-x} \mathrm{Sb}_{x} \mathrm{Te}_{3}$ slab. (a) Microarea XRD patterns. (b) Digital image of the annealed HTP slab. (c) Lattice parameters of the correlated micro regions presented in (a). 
a

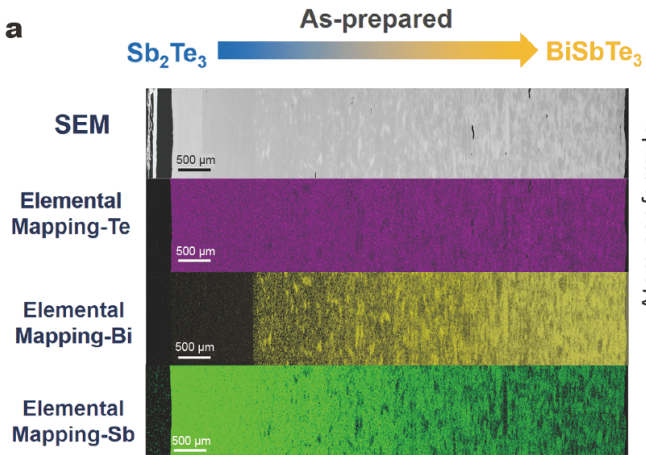

b

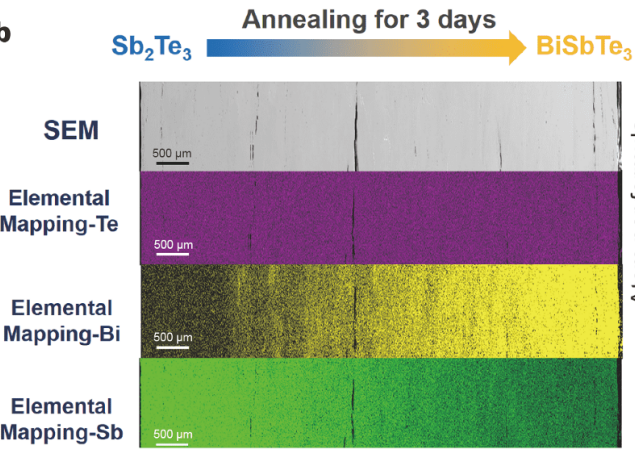

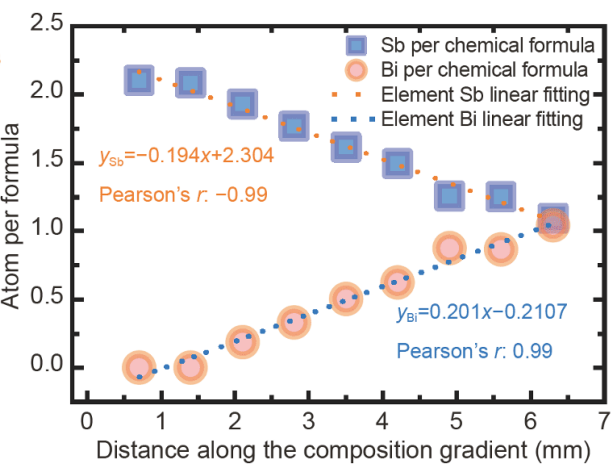

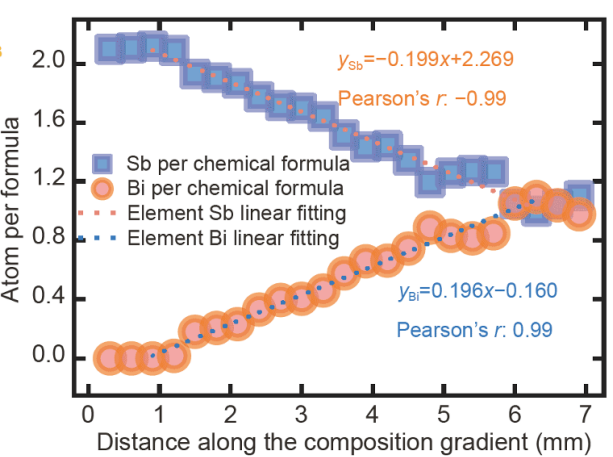

Figure 4 SEM-EDX elemental mappings for the HTP thin slab of $\mathrm{Bi}_{2-x} \mathrm{Sb}_{x} \mathrm{Te}_{3}$ with different annealing times. (a) As-prepared sample. (b) The sample annealed for 3 days.

coefficient distribution of the HTP $\mathrm{Bi}_{2-x} \mathrm{Sb}_{x} \mathrm{Te}_{3}$ thin slab shows a clear layered feature despite that it changes gradually along the composition gradient. To eliminate the error caused by the rough surface of the slab, the Seebeck coefficients of per column (along the longitudinal direction of the HTP thin slab) have been averaged (the right panel of Fig. $5 \mathrm{a}-\mathrm{c}$ ). It is worth noting that the Seebeck coefficient is very sensitive to the sample composition, leading to a sharper change around the interface' of different layers. This indicates the composition distribution of the HTP thin slab is not as continuous as expected, especially at the junction position of different layers which has been formed during the preparation of the raw material bar, and thus the HTP sample is denoted as a bulk sample with quasi-continuous composition. For the as-prepared $\mathrm{Bi}_{2-x} \mathrm{Sb}_{x} \mathrm{Te}_{3}$ sample, the average Seebeck coefficient increases from 95 to $155 \mu \mathrm{V} \mathrm{K}^{-1}$ along the composition direction of increasing Bi content. However, as shown in the right panel of Fig. 5a, the Seebeck coefficient of the as-prepared sample does not increase monotonically along the transverse direction of the HTP thin slab and significant fluctuations can be clearly observed. This implies that the as-prepared sample does not achieve a quasi-continuous composition distribution, which agrees well with the above SEM-EDX results. In contrast, the fluctuation of the Seebeck coefficient decreases after annealing, and the Seebeck coefficient of the sample annealed for 5 days shows nearly a quasi-continuous variation along the transverse direction of the HTP thin slab. However, the rear half of the average Seebeck coefficient $v s$. thin-slab position curve becomes almost a linear line after prolonging the annealing time to 5 days (the right panel of Fig. 5c), indicating that prolonging the annealing time promotes the diffusion of $\mathrm{Bi}$ and nearly homogeneous solid solution is formed for the regions with higher Bi content. Combining the Seebeck coefficient distribution measured by the PSM with the composition distribution determined by SEM-EDX, the $\mathrm{Bi}$ content dependent Seebeck coefficient of $\mathrm{Bi}_{2-x} \mathrm{Sb}_{x} \mathrm{Te}_{3}$ is obtained, as shown in Fig. 6. Thus, the maximum Seebeck coefficient around $155 \mu \mathrm{V} \mathrm{K}{ }^{-1}$, corresponding to the sample composition $\mathrm{BiSbTe}_{3}$, is screened out by our HTP experiments. Our Bi-content-dependent Seebeck coefficient for $\mathrm{Bi}_{2-x} \mathrm{Sb}_{x} \mathrm{Te}_{3}$ agrees well with the reported result in literature [33], verifying the reliability of our HTP screening strategy. For the $\mathrm{Bi}_{2} \mathrm{Te}_{3-x} \mathrm{Se}_{x}$ system, the Seebeck coefficient distribution displays similar layer feature (Fig. S6). A maximum Seebeck coefficient of about $-260 \mu \mathrm{V} \mathrm{K}^{-1}$ is obtained for the composition $\mathrm{Bi}_{2} \mathrm{Te}_{2.1} \mathrm{Se}_{0.9}$. 

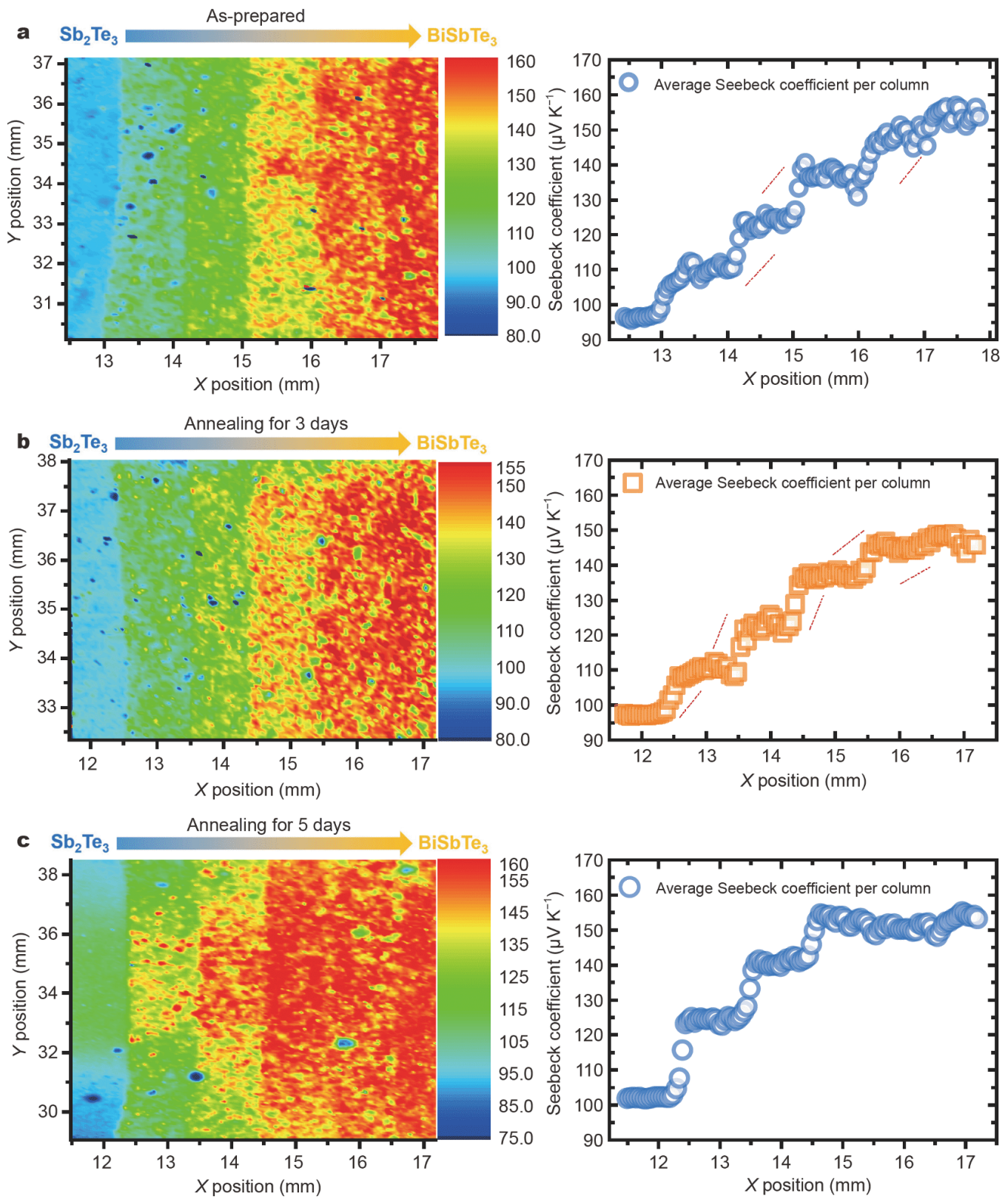

Figure 5 Seebeck coefficient distribution of the HTP $\mathrm{Bi}_{2-x} \mathrm{Sb}_{x} \mathrm{Te}_{3}$ thin slab. (a) As-prepared sample; (b) the sample annealed for 3 days; (c) the sample annealed for 5 days. The corresponding right panel shows the average Seebeck coefficient along the longitudinal direction of the HTP thin slab.

To screen out the optimum composition with the best thermoelectric performance, it is also necessary to measure the thermal conductivity which is essential for evaluating the thermoelectric properties. However, the commonly used thermal conductivity measurement methods (e.g., the laser flash technique) are mainly concentrated on the homogeneous sample. Present available methods are not suitable to characterize the thermal conductivity distribution of a bulk sample. In order to obtain the thermal property distribution of our HTP sample with quasi-continuous composition, a homemade thermal performance infrared screening system was developed. This apparatus is comprised of a rapid-heating unit and an infrared camera (Fig. S7). The HTP thin slab can be loaded into the heating chamber and positioned at the uniform temperature zone with a size of $22 \mathrm{~mm} \times$ $22 \mathrm{~mm}$. This very small uniform temperature zone ensures the precise heating temperature of the sample. 


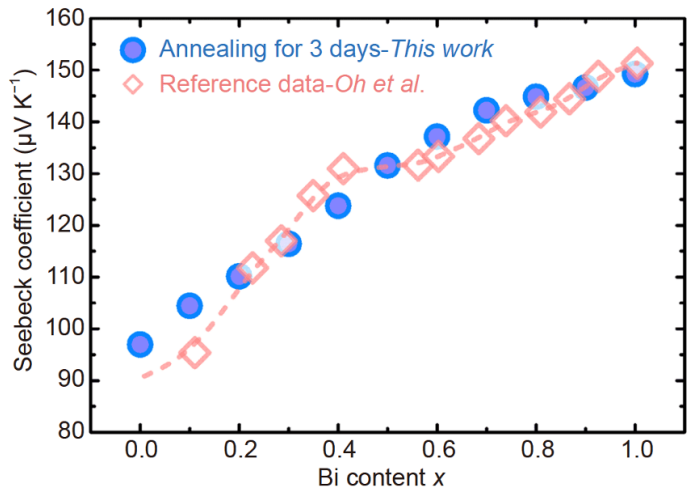

Figure 6 Bi-content-dependent Seebeck coefficient of the $\mathrm{Bi}_{2-x} \mathrm{Sb}_{x} \mathrm{Te}_{3}$ alloy. Reported data in literature [33] are included for comparison.

During the measurement, the bottom surface of the thin slab was rapidly heated to a given temperature in vacuum and held at this temperature, the temperature distribution at a certain time as well as the temperature change of a certain microarea of the top surface was monitored by the infrared camera. Theoretically, the thermal conductivity can be calculated based on the time-dependent tem- perature change. But this calculation requires the use of complex heat transfer theory and consideration of many measurement details, which is currently under development. Fortunately, it is very easy to know only the relative magnitude of thermal conductivity, which is directly reflected by the temperature distribution of the top surface of the HTP thin slab. Under the experimental conditions set above, the microarea/spot with lower temperature means that its thermal conductivity is also lower, and vice versa. In this work, the bottom surface of the HTP thin slab was heated to $100^{\circ} \mathrm{C}$ at the heating rate of $100^{\circ} \mathrm{C} \mathrm{min}^{-1}$. Fig. $7 \mathrm{a}$ shows the time dependent temperature variation of 12 marked regions for the $\mathrm{Bi}_{2-x} \mathrm{Sb}_{x} \mathrm{Te}_{3}$ slab annealed for 3 days. As shown in Fig. $7 \mathrm{~b}$, different regions of the top surface show different temperatures due to the quasi-continuous composition distribution, implying that the thermal conductivity varies with the sample composition. For both annealed and asprepared samples (Fig. S8), the region 12 shows lower surface temperature, which means lower thermal conductivity of that region. According to the SEM-EDX result, the composition of region 12 is close to $\mathrm{BiSbTe}_{3}$,
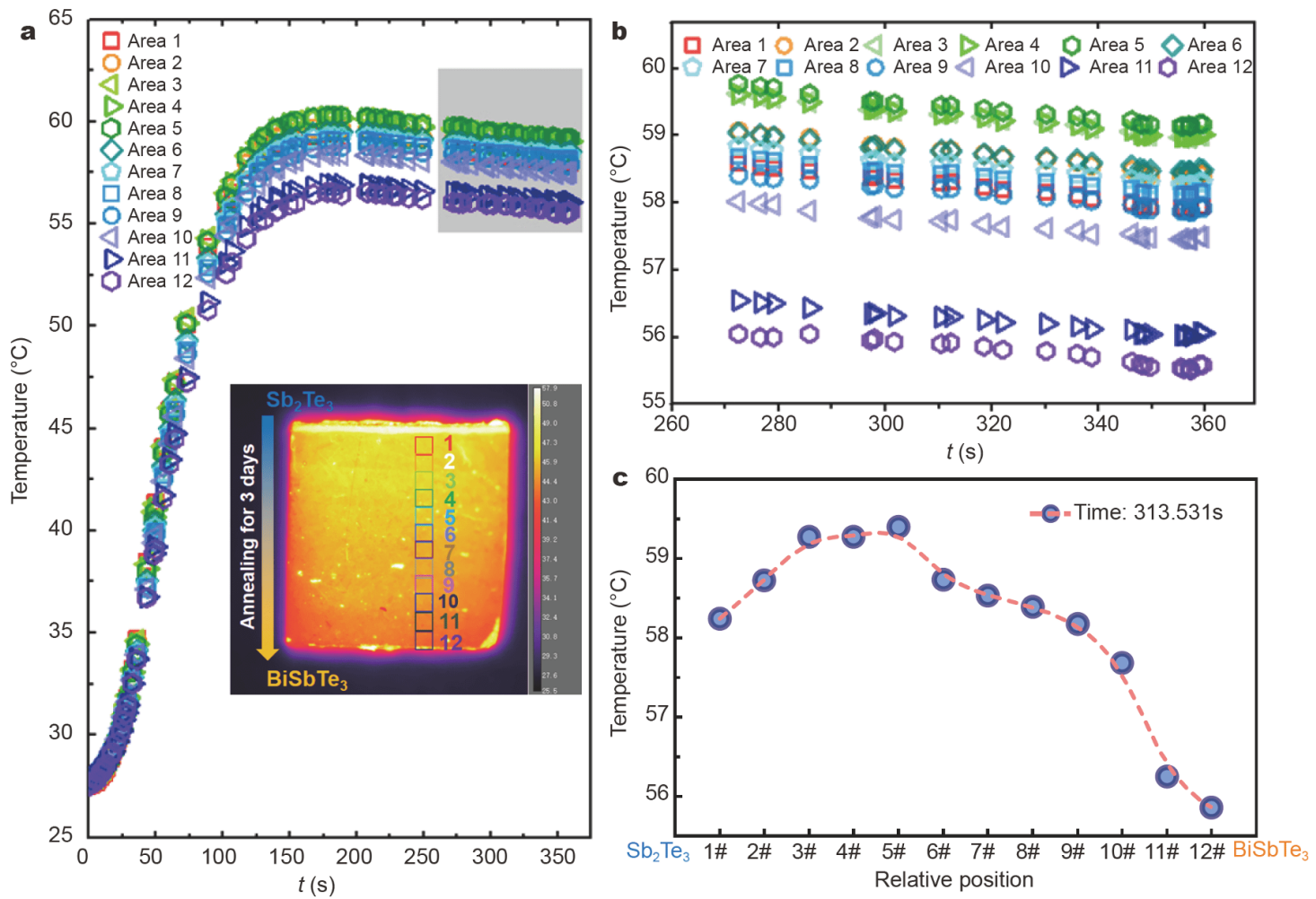

Figure 7 Top-surface temperature distribution of the $\mathrm{HTP} \mathrm{Bi}_{2-x} \mathrm{Sb}_{x} \mathrm{Te}_{3}$ thin slab annealed for 3 days. (a) The time-dependent temperature change of the marked regions shown in the inset of (a). The inset of (a) shows the image of the HTP thin slab captured by the infrared camera at $t=313.53 \mathrm{~s}$ (the corresponding bottom-surface temperature of the thin slab is $\sim 100^{\circ} \mathrm{C}$ ). (b) The enlarged view of the marked area in (a) by the grey square. (c) The temperature of the marked regions at $t=313.53 \mathrm{~s}$. 
suggesting the $\mathrm{BiSbTe}_{3}$ has the lowest thermal conductivity in the $\mathrm{Bi}_{2-x} \mathrm{Sb}_{x} \mathrm{Te}_{3}$ alloy with $x=1-2$ (Fig. 7c). Based on our HTP experiments, the target composition $\mathrm{BiSbTe}_{3}$ is screened out because it shows not only the largest Seebeck coefficient but also the lowest thermal conductivity, which is in good agreement with the literature report [33].

To illustrate the efficiency of our HTP strategy, the time taken by each process of the HTP experiment is summarized in the Sankey diagram. In this work, the machine time is defined as the time that the instrument is running. Human time refers specifically to the time required for a person to present and be occupied by the sample or machine. The melting and annealing time of the sample in the furnace as well as the time for preparing precursor materials are not counted. As shown in Fig. 1, it takes only $1140 \mathrm{~min}$ to screen out the target composition with

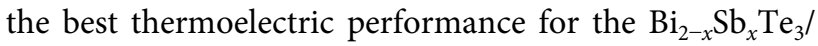
$\mathrm{Bi}_{2} \mathrm{Te}_{3-x} \mathrm{Se}_{x}$ system, and the labor time is greatly reduced to only $14.5 \%$ of the total experimental time.

In the full-chain HTP strategy, for proving the practicality of the HTP method, we chose the well-researched solid solution systems $\left(\mathrm{Bi}_{2-x} \mathrm{Sb}_{x} \mathrm{Te}_{3}\right.$ and $\left.\mathrm{Bi}_{2} \mathrm{Te}_{3-x} \mathrm{Se}_{x}\right)$. Even the sample forms a new compound with a certain composition, it could also be screened out based on our HTP method. On the one hand, it is possible to find the existence of new phases with the structure identification and composition analysis by XRD and SEM-EDX. On the other hand, the new phase can also be distinguished with electrical scanning and thermal properties mapping due to the difference in thermoelectric properties among the phases.

Here, we presented the HTP strategy in detail with each process. But it is not necessary to complete all the procedures in the practical experiments. The crucial processes should be selected as efficient as possible on screening the target composition with high thermoelectric performance. The HTP sample preparation process is indispensable, but HTP characterization can be chosen according to the actual needs of practical experiments. For example, it is not essential to analyze the composition and structure of the whole HTP sample in the initial stage and we can only concentrate on the microarea with high thermoelectric performance. The simplified process we recommend is as following: firstly, HTP samples are fabricated with quasi-continuous composition with our HTP preparation method. Secondly, the electrical and thermal performances of the samples are rapidly characterized by PSM and thermal performance infrared screening system. The target area with the highest ther- moelectric performance can be screened out. Finally, the crystal structure and composition of the target area will be determined by microarea XRD and SEM-EDX. In this way, we can only focus on the target area of optimum thermoelectric performance and it will effectively shorten the time of phase identification and composition analysis to screen out the thermoelectric materials.

\section{CONCLUSIONS}

In summary, we establish a HTP experimental technique for the screening of high-performance thermoelectric materials based on a bulk sample with quasi-continuous composition. For the preparation of the HTP bulk sample, a raw material bar with gradient composition was first prepared by a homemade automatic powder feeding system, and then the bulk sample with quasi-continuous composition for the measurement of composition and thermoelectric properties distribution was obtained by SPS sintering and subsequent annealing the raw material bar. The Seebeck coefficient and thermal conductivity distributions were characterized by the PSM and selfdeveloped thermal performance infrared screening system, respectively. The above HTP experiments were successfully realized for the $\mathrm{Bi}_{2-x} \mathrm{Sb}_{x} \mathrm{Te}_{3}$ and $\mathrm{Bi}_{2} \mathrm{Te}_{3-x} \mathrm{Se}_{x}$ systems. The target composition of $\mathrm{BiSbTe}_{3}$ with the largest Seebeck coefficient and lowest thermal conductivity near room temperature was screened out, demonstrating the practicability of our HTP experiment. In addition, the total time and labor time spent in finding the target material were greatly reduced, displaying the high efficiency of our HTP strategy.

Received 23 September 2020; accepted 20 November 2020; published online 4 February 2021

1 Potyrailo R, Rajan K, Stoewe K, et al. Combinatorial and highthroughput screening of materials libraries: Review of state of the art. ACS Comb Sci, 2011, 13: 579-633

2 Luo J, He SY, Li ZL, et al. Progress on high-throughput synthesis and characterization methods for thermoelectric materials. J Inorg Mater, 2019, 34: 247

3 Maier WF, Stöwe K, Sieg S. Combinatorial and high-throughput materials science. Angew Chem Int Ed, 2007, 46: 6016-6067

4 Jain A, Ong SP, Hautier G, et al. Commentary: The materials project: A materials genome approach to accelerating materials innovation. APL Mater, 2013, 1: 011002

5 Niu X, Gielen F, Edel JB, et al. A microdroplet dilutor for highthroughput screening. Nat Chem, 2011, 3: 437-442

6 Yan J, Gorai P, Ortiz B, et al. Material descriptors for predicting thermoelectric performance. Energy Environ Sci, 2015, 8: 983-994

7 Beal MS, Hayden BE, Le Gall T, et al. High throughput methodology for synthesis, screening, and optimization of solid state lithium ion electrolytes. ACS Comb Sci, 2011, 13: 375-381

8 Otani M, Lowhorn ND, Schenck PK, et al. A high-throughput 
thermoelectric power-factor screening tool for rapid construction of thermoelectric property diagrams. Appl Phys Lett, 2007, 91: 132102

9 Raccuglia P, Elbert KC, Adler PDF, et al. Machine-learning-assisted materials discovery using failed experiments. Nature, 2016, 533: $73-76$

10 Carrete J, Li W, Mingo N, et al. Finding unprecedentedly lowthermal-conductivity half-heusler semiconductors via highthroughput materials modeling. Phys Rev X, 2014, 4: 011019

11 Varley JB, Miglio A, Ha VA, et al. High-throughput design of nonoxide p-type transparent conducting materials: Data mining, search strategy, and identification of boron phosphide. Chem Mater, 2017, 29: 2568-2573

12 Zhu H, Hautier G, Aydemir U, et al. Computational and experimental investigation of $\mathrm{TmAgTe}_{2}$ and $\mathrm{XYZ}_{2}$ compounds, a new group of thermoelectric materials identified by first-principles high-throughput screening. J Mater Chem C, 2015, 3: 10554-10565

13 Xi L, Pan S, Li X, et al. Discovery of high-performance thermoelectric chalcogenides through reliable High-throughput material screening. J Am Chem Soc, 2018, 140: 10785-10793

14 Senkan SM. High-throughput screening of solid-state catalyst libraries. Nature, 1998, 394: 350-353

15 Xiang XD. Combinatorial materials synthesis and screening: An integrated materials chip approach to discovery and optimization of functional materials. Annu Rev Mater Sci, 1999, 29: 149-171

16 Gómez-Bombarelli R, Aguilera-Iparraguirre J, Hirzel TD, et al. Design of efficient molecular organic light-emitting diodes by a high-throughput virtual screening and experimental approach. Nat Mater, 2016, 15: 1120-1127

17 You L, Li Z, Ma Q, et al. High thermoelectric performance of Cudoped $\mathrm{PbSe}-\mathrm{PbS}$ system enabled by high-throughput experimental screening. Research, 2020, 2020: 1-8

18 de Pablo JJ, Jackson NE, Webb MA, et al. New frontiers for the materials genome initiative. npj Comput Mater, 2019, 5: 41

19 Ortiz BR, Adamczyk JM, Gordiz K, et al. Towards the highthroughput synthesis of bulk materials: Thermoelectric PbTePbSe-SnTe-SnSe alloys. Mol Syst Des Eng, 2019, 4: 407-420

20 Xiang XD, Sun X, Briceño G, et al. A combinatorial approach to materials discovery. Science, 1995, 268: 1738-1740

21 Wambach M, Stern R, Bhattacharya S, et al. Unraveling self-doping effects in thermoelectric TiNiSn half-heusler compounds by combined theory and high-throughput experiments. Adv Electron Mater, 2016, 2: 1500208

22 Mao SS. High throughput growth and characterization of thin film materials. J Cryst Growth, 2013, 379: 123-130

23 Hedegaard EMJ, Mamakhel AAH, Reardon H, et al. Functionally graded $(\mathrm{PbTe})_{1-x}(\mathrm{SnTe})_{x}$ thermoelectrics. Chem Mater, 2018, 30: 280-287

24 Hedegaard EMJ, Johnsen S, Bjerg L, et al. Functionally graded
$\mathrm{Ge}_{1-x} \mathrm{Si}_{x}$ thermoelectrics by simultaneous band gap and carrier density engineering. Chem Mater, 2014, 26: 4992-4997

25 Ferreres XR, Aminorroaya Yamini S. Rapid fabrication of diffusion barrier between metal electrode and thermoelectric materials using current-controlled spark plasma sintering technique. J Mater Res Tech, 2019, 8: 8-13

26 Ikeda $\mathrm{T}$, Iwanaga $\mathrm{S}, \mathrm{Wu} \mathrm{H}$, et al. A combinatorial approach to microstructure and thermopower of bulk thermoelectric materials: the pseudo-ternary $\mathrm{PbTe}-\mathrm{Ag}_{2} \mathrm{Te}_{-}-\mathrm{Sb}_{2} \mathrm{Te}_{3}$ system. J Mater Chem, 2012, 22: 24335

27 Zhao JC, Jackson MR, Peluso LA, et al. A diffusion multiple approach for the accelerated design of structural materials. MRS Bull, 2002, 27: 324-329

$28 \mathrm{Pu}$ HY, Xie RQ, Peng Y, et al. Accelerating sample preparation of graded thermoelectric materials using an automatic powder feeding system. Adv Manuf, 2019, 7: 278-287

29 Zhao JC. Reliability of the diffusion-multiple approach for phase diagram mapping. J Mater Sci, 2004, 39: 3913-3925

30 Bergum K, Ikeda T, Jeffrey Snyder G. Solubility and microstructure in the pseudo-binary $\mathrm{PbTe}-\mathrm{Ag}_{2} \mathrm{Te}$ system. J Solid State Chem, 2011, 184: 2543-2552

31 Wei C, Antolin N, Restrepo OD, et al. A general model for thermal and electrical conductivity of binary metallic systems. Acta Mater, 2017, 126: 272-279

32 Huxtable S, Cahill DG, Fauconnier V, et al. Thermal conductivity imaging at micrometre-scale resolution for combinatorial studies of materials. Nat Mater, 2004, 3: 298-301

33 Oh T. Thermoelectric properties of the hot-pressed $(\mathrm{Bi}, \mathrm{Sb})_{2}(\mathrm{Te}, \mathrm{Se})_{3}$ alloys. Scripta Mater, 2000, 42: 849-854

Acknowledgements This work was supported by the National Key Research and Development Program of China (2018YFB0703600 and 2018YFA0702100), and the National Natural Science Foundation of China (51772186, 51632005 and 51371194).

Author contributions Luo J designed the research; He S and Yang Y prepared the HTP samples; Li Z performed the SEM characterization; Li $Z$, Zhang $J$ and $\mathrm{He} S$ conducted the microarea XRD measurement; He $S$ and Zhang J measured the electrical and thermal properties of the HTP samples; He S, Yang Y, Zhang W and Luo J analyzed the experimental data; He $\mathrm{S}$ and Luo J prepared the manuscript and all authors commented on the manuscript.

Conflict of interest The authors declare that they have no conflict of interest.

Supplementary information online version of the paper.
Supporting data are available in the 


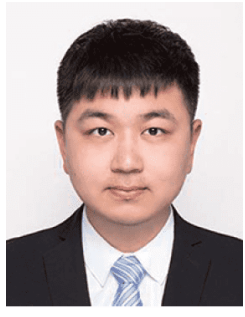

Shiyang He received his BSc degree in materials science and engineering from Zhengzhou University in 2017, and Master degree from Shanghai University under the supervision of Prof. Jun Luo in 2020. His research focuses on flexible thermoelectric materials and high-throughput experimental screening of thermoelectric materials.

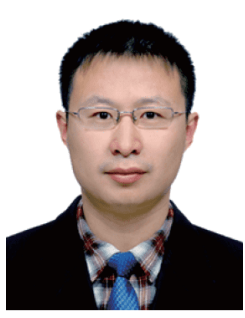

Yang Yang received his $\mathrm{PhD}$ degree in 2015 from Ritsumeikan University (Kyoto, Japan). He is a lecturer at the School of Mechatronic Engineering and Automation, Shanghai University. His research interests include soft robots, mobile robots, and terramechanics.

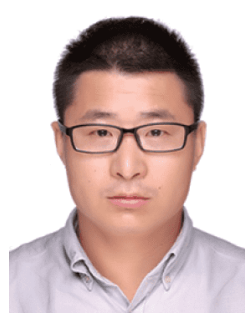

Jun Luo received his BSc and MSc degrees from the University of Science and Technology Beijing in 1999 and 2002, respectively, and a $\mathrm{PhD}$ degree in condensed matter physics from the Institute of Physics, Chinese Academy of Sciences in 2005. $\mathrm{He}$ is currently a professor of Shanghai University. His research focuses on thermoelectric materials.

\section{一种通用的块体热电材料高通量实验篮选策略}

何世洋 ${ }^{1 \dagger}$, 杨扬 $^{2 \dagger}$, 李志立 ${ }^{1}, 弓^{\circ}$ 继业 $^{1}$, 王晨阳 ${ }^{1}$, 张文清 ${ }^{3}$, 骆军 ${ }^{1,4^{*}}$ 摘要 高通量实验对加速先进材料的发现起着关键作用, 但是高通 量制备和表征, 尤其是块体样品的高通量制备和表征非常困难. 本 文报道了一种新型通用的高性能块体热电材料高通量篎选策略. 该全链条高通量实验涵盖准连续成分大块样品的快速制备, 微区 物相和结构分析, 以及样品成分、电学和热学输运性质的空间分 布表征. 我们通过该高通量实验方法快速制备出了成分准连续分 布的 $\mathrm{Bi}_{2-x} \mathrm{Sb}_{x} \mathrm{Te}_{3}(x=1-2)$ 和 $\mathrm{Bi}_{2} \mathrm{Te}_{3-x} \mathrm{Se}_{x}(x=0-1.5)$ 块体样品. 后续 的高通量实验表征证实我们成功䇥选出了具有最佳 $\mathrm{Sb} / \mathrm{Bi}$ 和 $\mathrm{Te} / \mathrm{Se}$ 成分比的目标热电材料，表明该高通量实验技术在加速新型高性 能热电材料的探索方面十分有效. 